\title{
A Collaborative Vacuum Tool for Humans and Robots
}

\author{
Wilson Hernandez, Alvaro Hilarion, and Carol Martinez ${ }^{(凶)}$ \\ Department of Industrial Engineering, School of Engineering, \\ Pontificia Universidad Javeriana, Bogotá, Colombia \\ carolmartinez@javeriana.edu.co
}

\begin{abstract}
This paper presents the design and implementation of a tool for Human-Robot collaborative tasks. Industry 4.0 proposes a new scenario where robots can safely work in direct cooperation with humans, within a defined workspace. These robots are called collaborative robots or COBOTS. In this paper, we propose a tool that can be used by a robot and/or an operator for pick and place tasks. The tool was designed to meet certain criteria for the robot, so that it can identify it, hold it, and perform the task. Additionally, it was designed to comply with ergonomic parameters for the human operator. A prototype of the tool was printed with a FusedForm 3D printer. This prototype was used for a test where a robot and an operator will use the tool for picking plastic bottles from a worktable. An UR3 robot was used in the test. It was equipped with a camera in charge of identifying and estimating the position of the tool using ArUco codes placed on the tool. Results show the functionality of the tool for both the robot and the human operator. Additionally, ergonomic tests provided insights to improve the handling system of the tool for the human operator.
\end{abstract}

\section{Introduction}

Collaborative robots work in spaces immersed with humans. They should be safe, easy to use, and flexible in terms of the tasks they conduct [1]. One of the most important parts of the robots is its end effector. It allows the robot to conduct different tasks. End effectors can be found in different styles. Suction cups, grippers (typically two or more fingers), spot-welding tools, paint sprayer, or almost anything else that can meet the application needs. Most of the time, collaborative robotics has focused on the robot side. As Kieffer mentions in [4]: "End users want a collaborative robot application. You can not make that if only the robot is collaborative". Additionally in [13], it is mentioned that it is important to develop collaborative grippers, taking into account that "they might come into contact with the people". They must be carefully designed, so that the tool exchange time should be short to avoid affecting production requirements. Currently, the exchange of tools is conducted in several ways [9]. The first mode is the manual exchange. It is the simplest mode, but it has disadvantages such as 
its high costs and that the operator must enter the workspace of the robot every time the tool has to be changed. The second mode is automated exchange. The exchange is done by commands using the robots control system. The automated gripper exchanges can be divided into three groups:

- multi launching grippers

- whole gripper exchange.

- clamping pads.

An example of an automatic exchanger is the one developed by Kuka [9]. It is a multifunctional end effector (MFEE) for 6 different tools, such as spindle, for sealant applications, hammer modules, and milling, among others (Fig. 1, left image). On the other hand, Fig. 1 right image shows an example of the MC-10 manual exchanger developed by ATI Industrial Automation [2]. It allows the connection of different tools in a simple way.
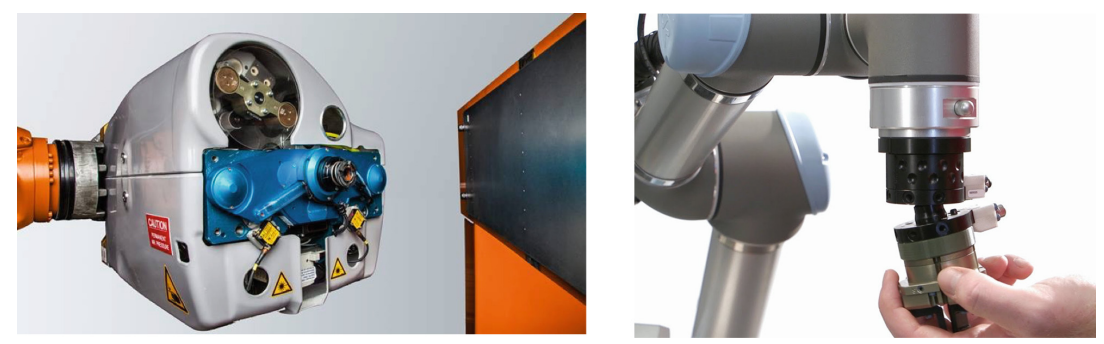

Fig. 1. Commercial exchanger. On the left, the automatic multifunctional end effector (MFEE) developed by Kuka [9]. On the right, the MC-10 Manual Tool Changer developed by ATI [2].

Another type of commercial grippers are the adaptive grippers. They have the advantage that they adapt to different size and shapes using simple mechanisms and simple control [9]. Some examples are shown in Fig. 2. The SRDK-UR from Universal Robots (Fig. 2, left image) is the first customizable grip system that imitates the human hands in tasks that require flexibility, dexterity, and precision [11]. The FlexShape Gripper by Festo (Fig. 2, right image) uses the chameleon's tongue principle to grip. It can grip different types of objects regardless their shape.

Industry 4.0. proposes a new role for industrial robots where robots do not substitute humans, but instead they work side by side with them [12]. Based on this, one can think on scenarios where robots and humans could share the same tools, without the need to buy and place expensive additional mechanisms for the robot every time we need the robot to handle specific objects. To the best of the author's knowledge, there have not been works presented in the literature focused on this kind of tools. 

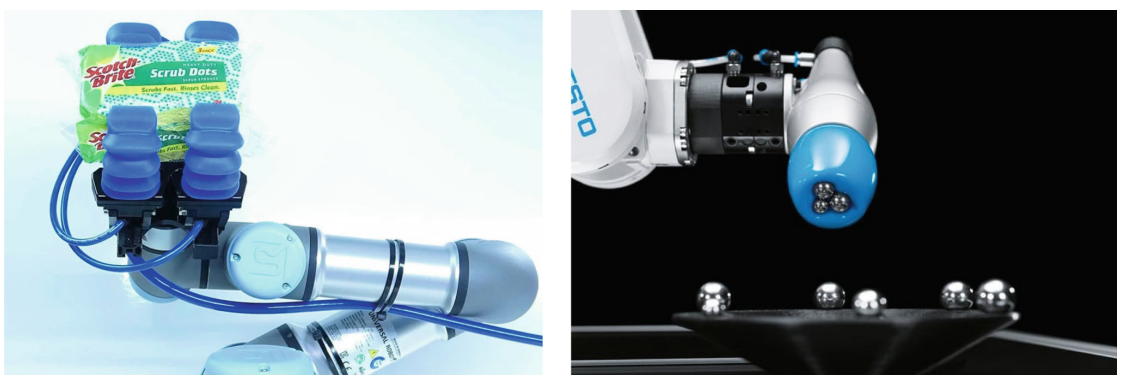

Fig. 2. Commercial grippers. On the left, The SRDK-UR from Universal Robots. It imitates the human hands in terms of flexibility, dexterity, and precision [11]. On the right, the FlexShape Gripper by Festo which can grip different types of objects regardless their shape.

The design and development of the tool is part of the PIR (Perception for Industrial Robots) project from the Pontificia Universidad Javeriana Bogota (PUJ) [6]. This project is focused on characterizing and simulating the application of an industrial robot for waste separation tasks and conducting research on the technical aspects of the collaborative and flexibility concepts of COBOTs. In this article, we propose a vacuum tool that can be used for picking plastics bottles in the bottle classification task conducted in the Alquería recycling center in Bogotá. There, tasks are conducted manually, thus this being a perfect scenario for testing the collaboration between humans and robots (see Fig. 3).
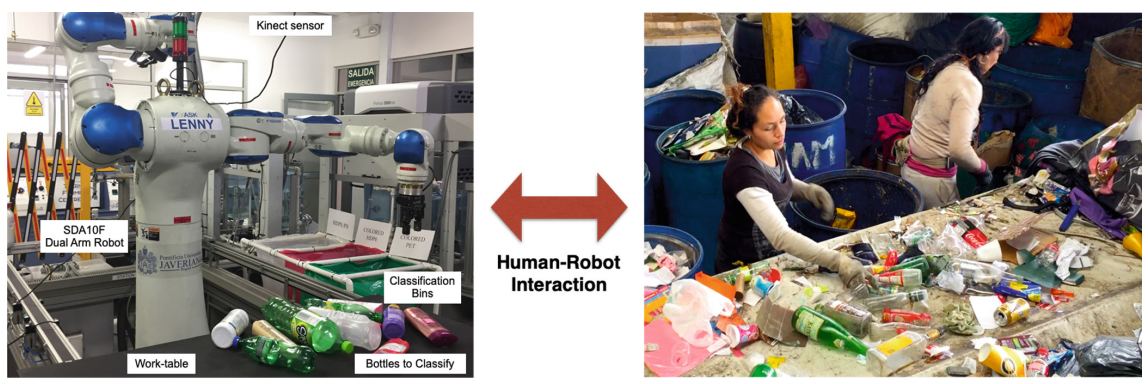

Fig. 3. Human-Robot Interaction proposed for waste classification tasks at the Alquería recycling center [10].

The Alquería recycling center is responsible for separating color High Density Polyethylene (HDPS), High Density Polyethylene/Polystyrene (HDPS/PS), green polyethylene terephthalate (PET), and transparent PET, among others. Due to the wide variety of bottles that arrive to the recycling center, e.g. different sizes; the robot grippers, in some cases, are not the appropriate tools for grasping the containers. Therefore, within the project we propose a tool magazine that will be in the robot's workspace and will be accessible either by the 
robot or the operator, if required. In the magazine, different vacuum tools of different sizes can be available.

The paper is organized as follows, Sect. 2 shows the designed criteria considered for designing the tool. Section 3 presents the prototype. Section 4 describes the tests carry out to evaluate the tool, and Sect. 5 presents the conclusion and the direction of future work.

\section{Design Criteria}

Grippers are tools that are only designed for robots. However, the proposed tool targets both robots and humans.

To meet the human's specifications, anthropometric measurements of the hand of adults (shown in Fig.4) and ergonomic parameters that are based on the type of activity to be developed, were considered. Table 1 summarizes the parameters considered when designing the tool from the human point of view, taking into account the application (pick and place of plastic bottles).

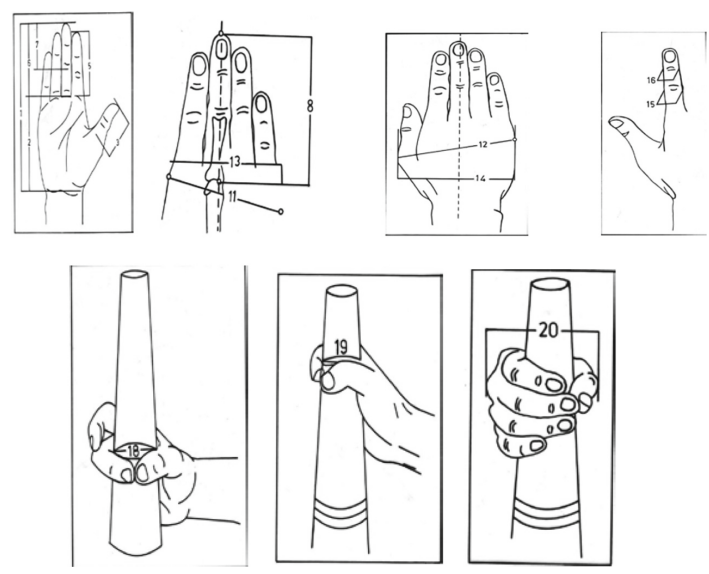

Fig. 4. Anthropometric measures of the hand of an adult for holding object [8].

Additional aspects were considered in the design of the tool:

- The tool should encourage the use of both hands, to help mitigating the problems of left-handed people and fatigue when the dominant hand is fatigued. For more than $90 \%$ of users, the dominant hand is the right one.

- The design should follow strictly the design criteria, this because an intense compression of the tool leads to the inflammation of the tendon sheaths, compromising blood supply and leading to an excessive compression of critical nerves [14].

- The weight of the tool should not exceed $3 \mathrm{Kg}$. This to comply with the limitations stablished by the ISO 11228 about manual handling [3]. 
Table 1. Ergonomic parameters considered for the design of the tool.

\begin{tabular}{l|l}
\hline Factor & Parameters \\
\hline Activity & $\begin{array}{l}\text { Type of task. Pick and place } \\
\text { Work environment. Workers are standing up in } \\
\text { an 8-h shift. The operator must pick and place } \\
\text { the bottles in a basket that is close to the table }\end{array}$ \\
\hline Postural and biomechanical analysis & $\begin{array}{l}\text { Body posture. Standing when handling plastic } \\
\text { bottles } \\
\text { The tool } \\
\text { Hand posture and hand-wrist movement. } \\
\text { Natural posture of the wrist }\end{array}$ \\
$\begin{array}{l}\text { Shape of the tool. The handle must provide } \\
\text { maximum contact between the tool and the skin } \\
\text { (or glove). In general, its transverse section } \\
\text { should be cylindrical, flat, or elliptical [7] } \\
\text { Dimension and weight. The user must be able } \\
\text { to use the tool with one hand. Ideal weights of } \\
\text { tools operated by the hand-forearm segment is } \\
0.9-1.5 \text { Kg. Length of the handle for force tools: } \\
100-125 \text { mm } \\
\text { Surface of the handle. Should ensure a good } \\
\text { grip on a handle. There must be enough friction } \\
\text { between the hand and the handle. Handles made } \\
\text { of plastic or composite rubber are recommended }\end{array}$ \\
\hline
\end{tabular}

In addition to the human factor, the robot's gripping system was considered when designing the tool. In this study, the hardware shown in Fig. 5 was used. It is comprised of the UR3 robot from Universal robots, and the two fingers gripper from Robotiq. The parameters of the robot that are considered for the design of the tool are shown in Table 2. In addition to those criteria, the tool
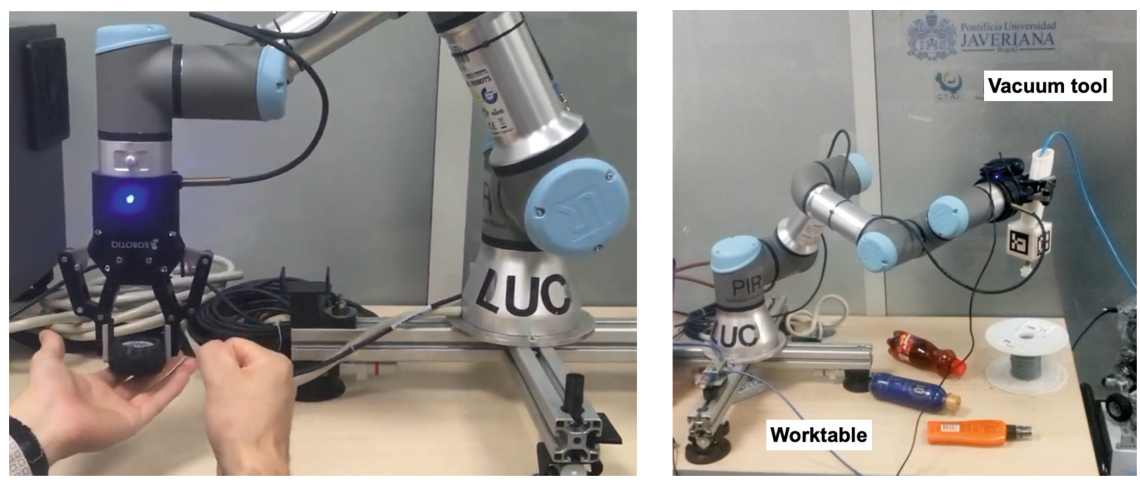

Fig. 5. Testbed. It is comprised of the UR3 collaborative robot from Universal robots equipped with the two fingers gripper from Robotiq. 
must be easily identified, so that the robot can automatically locate the tool when it needs it.

Table 2. Robot parameters

\begin{tabular}{l|l}
\hline Characteristics & Value \\
\hline Robot payload & $3 \mathrm{~kg}$ \\
\hline Friction grip payload & $3 \mathrm{~kg}$ \\
\hline Gripper weight & $1 \mathrm{~kg}$ \\
\hline Stroke & $50 \mathrm{~mm}$ \\
\hline Grip force & 60 to $130 \mathrm{~N}$ \\
\hline
\end{tabular}

\section{Prototype}

Figure 6 summarizes the criteria considered for designing the tool. As It is shown in the image, the tool was designed considering some criteria only for the human (body posture, hand posture, etc.); but additionally there are other criteria such as, shape, weight, and surface, that required to be evaluated for both the human and the robot.

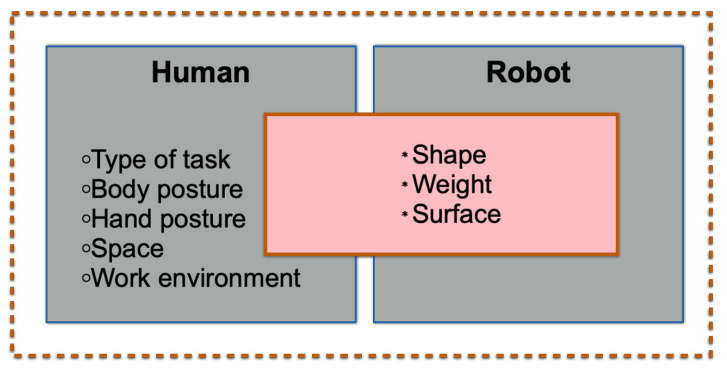

Fig. 6. Ergonomic and robot parameters for the design of the tool. Shape, Weight, and Surface criteria should comply the design criteria for both, the human and the robot.

Figure 7 shows the designed tool. The upper part (marked with number 1), correspond to the handle, where the operator and the robot will hold the tool. The lower part (marked with number 2) corresponds to a cube, where the suction cup will be placed in. The lower part was designed to allow the placement of visual markers on the tool (ArUco codes) for its automatic detection and identification.

The handle of the tool was designed with a length of $123 \mathrm{~mm}$ and a diameter of $38 \mathrm{~mm}$. In addition, the handle has an inclination of $12^{\circ}$, following ergonomics recommendations for manual tools. The tool will have also a button to allow manual activation of the vacuum. 

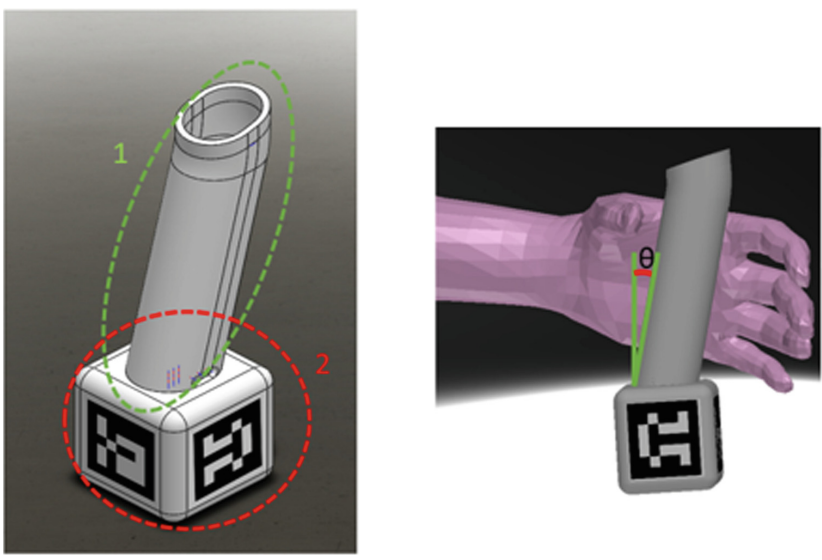

Fig. 7. Prototype of the tool. The upper part (number 1), correspond to the handle that will be shared by the robot and the operator. The lower part (number 2) will contain the succion cup and visual markers for automatic detection and identification of the tool.

\section{Test and Results}

Three different tests were conducted to analyze the design of the tool. The first one was focused on the robot; the second one, analyzing people's opinion about the tool; and the third one corresponds to a test where the robot and a person shared the tool.

\subsection{Robot}

In this test, the UR3 robot was used to automatically detect the tool and pick up 9 bottles of different sizes grouped as small, medium, and big (three bottles per size). This test was focused on verifying that the robot can pick up the tool and that it does not slip from the gripper's fingers (i.e. that the bottle does not fall). Figure 8 shows the robot and some images of the bottles the robot had to pick up.

The test consisted on commanding the robot to pick up the bottles. For each group of bottles (small, medium, and big), the test was conducted 10 times at 3 different speeds, low (10\% mx. robot's speed), medium 10 (50\% mx. robot's speed) and high (90\% mx. robot's speed). The maximum speed of the UR3 robot is $1 \mathrm{~m} / \mathrm{s}$. If the bottles of each group were successfully picked up, the test was considered successful, otherwise failed.

The results of the test are summarized in Table 3. From this table, it can be seen that the system picked up all the small bottles and that the change of speed did not affect the results. On the other hand, the robot succeeded in $93 \%$ of the tests, when picking up the medium size bottles; and $83 \%$ of the tests, when picking up the big size bottles. Analyzing the results, the 7 trials when 

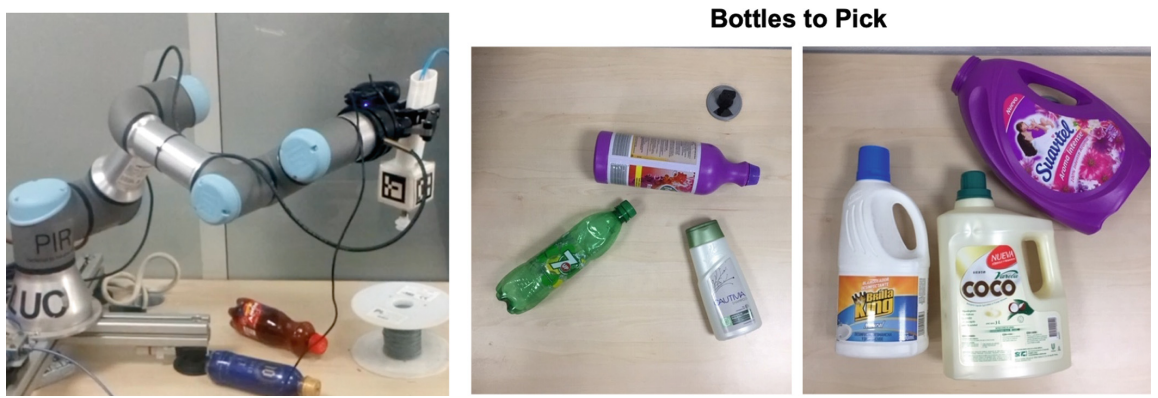

Fig. 8. Setup Test 1. The robot shown on the left image was commanded to pick up 3 bottles of each group 10 times at three different speeds (30 trials).

the robot failed carrying the bottles correspond to tests conducted at high speed and when the vacuum tool was far from the center of gravity of the bottle.

Table 3. Results Test 1. Robot picking up 3 bottles of each group 10 times at three different speeds (30 trials). If all the bottles of each group were successfully picked up, the test was considered successful, otherwise failed.

\begin{tabular}{l|l|l}
\hline Bottle's size & Successful trials & Failed trials \\
\hline Small & 30 & 0 \\
\hline Medium & 28 & 2 \\
\hline Big & 25 & 5 \\
\hline
\end{tabular}

\subsection{Ergonomic Test}

A test was carried out to analyze the people's opinion regarding the tool. Five persons where questioned about ergonomic and manipulation aspects of the proposed tool when using the tool for picking up the bottles. Each person handled 10 plastic bottles with the tool and answered the following survey. The answer for each question was limited to Good, Regular, or Bad.

1. Is the handle length enough for the size of your hand?

2. Is the handle diameter comfortable?

3. Is the weight of the tool considered to be adequate?

4. Is the position of the suction cup correct?

5. Is the position of the activation button of the suction cup correct?

Table 4 summarizes the results obtained in the survey. It shows the number of people whose answer coincided in each question. The following list summarizes the people's comments for each question. 
1. Handle length. People commented that the length of the tool was appropriate for their hands and they were able to manipulate the tool without problems. They recommended to improve the material of the handle to prevent it form slipping.

2. Handle diameter. People with small hands recommended to reduce the diameter of the handle to make it more comfortable for them.

3. Tool's weight. Everybody agreed that the weight was appropriate for manipulating the tool.

4. Suction cup. Most of them commented that it was difficult to see the suction cup.

5. Activation button. They agreed that the position of the activation button was correct, but that it required a lot of force to be activated.

Table 4. Results Test 2. The table summarizes the number of people whose answer coincided for each of the question related to ergonomic and manipulation aspects, when using the tool.

\begin{tabular}{l|l|l|l}
\hline \multirow{2}{*}{ Question } & \multicolumn{3}{l}{ Scores } \\
\cline { 2 - 4 } & Good & Regular & Bad \\
\hline 1 & 4 & 1 & 0 \\
\hline 2 & 3 & 2 & 0 \\
\hline 3 & 5 & 0 & 0 \\
\hline 4 & 1 & 4 & 0 \\
\hline 5 & 2 & 3 & 0 \\
\hline
\end{tabular}

\subsection{Human-Robot Test}

A final test was conducted to have both, the human and the robot using the tool for picking up plastic bottles. Figure 9 shows representative images from the test, where the human is manipulating the tool for picking up some bottles.

Figure 10 shows images when the robot was conducting the task. The tool was automatically detected and identified by a computer vision algorithm. ArUco markers located on the four sides of the cube were used to provide fast 3D pose estimations of the tool to the robot. With this markers, the human can place the tool in any orientation and the system can still detect the tool.

A video of the test can be seen in [5]. 

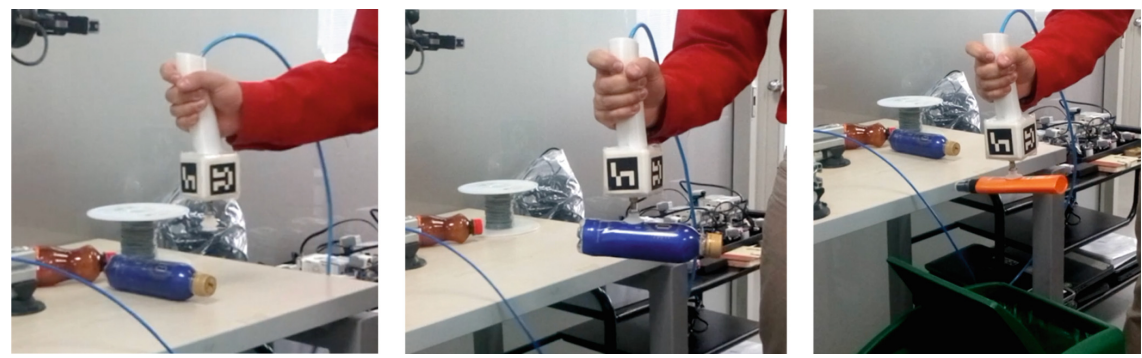

Fig. 9. Results Test 3 Human Manipulating the Tool. Representative images of the test when the human uses the tool for picking up some bottles.
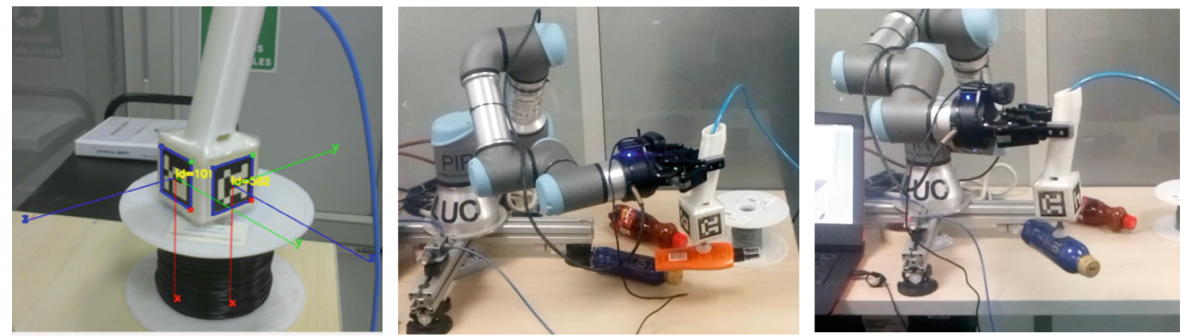

Fig. 10. Results Test 3 Robot Manipulating the Tool. Image on the left shows the computer vision algorithm the robot uses to detect and identify the tool. The other two images are representative images of the test when the robot picks up some bottles with the tool.

\section{Conclusions}

In this paper we presented the design of a vacuum tool that can be shared by humans and robots, for plastic classification tasks. Real tests were conducted, and the results showed that the robot was able to successfully pick up of small bottles at different speed. With the medium size and big size bottles, some of the bottles fell off when operating at high speed. However, it is important to mention that the weight of those bottles was greater than the weight the suction cup can handle.

From the results, we can say that the vacuum tool can be carry by the robot appropriately, since in none of the tests the tool fell off the robot. Future work is focused on improving ergonomic aspects of the tool, based on the suggestions provided by the people involved in the tests.

Acknowledgements. This work has been supported by the Pontificia Universidad Javeriana Bogota. Project 7697, PIR Perception for Industrial Robots. The authors would like to thank the CTAI for the support with the equipments. 


\section{References}

1. AER: Robotica colaborativa. https://www.aer-automation.com/mercados-emerge ntes/robotica-colaborativa/

2. ATI-Industrial-Automation: ATI tool changer products: MC-10 (2018). https:// www.ati-ia.com/products/toolchanger/QC.aspx?ID=MC-10

3. Becker, J.P.: Las normas iso 11228 en el manejo manual de cargas (2009). http:// www.semac.org.mx/archivos/congreso11/Pres09.pdf

4. Crowe, S.: 6 innovative robotic grippers lend a helping hand (2018). https://www. therobotreport.com/6-innovative-robotic-grippers-lend-helping-hand/

5. CTAI: A collaborative vacuum tool for humans and robots (2018). https://www. youtube.com $/$ watch? $\mathrm{v}=5$ tzehMjjQJQ

6. CTAI: Pir recycling (2018). https://www.youtube.com/watch?v=F76Pe-WkP3g\& $\mathrm{t}=2 \mathrm{~s}$

7. Ergonomia108: Diseño y seleccion de herramientas (2013). http://ergonomia108. blogspot.com/2013/10/23-diseno-y-seleccion-de-herramientas.html

8. Jain, S., Pathmanathan, G.: Importance of anthropometry for designing userfriendly devices: mobile phones. J. Ergon. (2012). https://doi.org/10.4172/21657556.1000109

9. Kerak, P., Holubek, R.: Automatic gripper exchange in intelligent manufacturing systems, pp. 1313-1314 (2011)

10. Lizarazo, N.B., Rico, D.G., Martinez, C., Atuesta, S.B.: Designing a framework to give perception capabilities to an industrial robot for waste separation tasks, p. 39 (2017)

11. Universal Robots: Efectores finales: Cobots con destreza humana (2018). https:// blog.universal-robots.com/es/efectores-finales-cobots

12. Universal Robots: La industria 4.0: Camino hacia la automatización y la vanguardia (2018). https://blog.universal-robots.com/es/industria-40

13. Schlichtb, L.B.T.: A statistical review of industrial robotic grippers, pp. 88-97 (2018). https://doi.org/10.1016/j.rcim.2017.05.007

14. de Seguridad e Higiene en el Trabajo (INSHT): Herramientas manuales. criterios ergonomicos y de seguridad para su seleccion (2016). https://www.insst.es/ InshtWeb/Contenidos/Documentacion/ 